https://doi.org/10.36909/jer.v9iICRIE.11667

\title{
Adaptive Real-Time Wavelet Denoising Architecture Based on Feedback Control Loop
}

\author{
Fars Samann*, Serwan Ali Bamerni*, Jeeman Ahmed Khorsheed* and Ahmed Khorsheed \\ Al-sulaifanie* \\ College of Engineering, University of Duhok, Kurdistan Region, Iraq
}

*Email: fars.samann@uod.ac; Corresponding Author.

\begin{abstract}
The discrete wavelet transform is commonly used as a denoising step for many applications, like biomedical applications which are usually suffering from low SNR of the recorded signal. However, the choice of appropriate threshold value for DWT coefficients plays significant role in reconstructing the denoised signal. This paper presents a design of real-time wavelet denoising architecture which is suitable for wide range of real-time denoising applications. In this design, an adaptive thresholding approach based on feedback control loop is proposed to make the architecture more applicable for real-time wavelet denoising. This thresholding method considers a noise level estimator module based on first detail coefficients level $d_{1}$ to calculate the unknown standard deviation of background noise. The proposed architecture is developed using MATLAB to simulate the suggested denoising method. The performance of the proposed denoising method is studied in terms of integral gain $G$ of feedback control and window size $M$ with respect to the improvement in SNR and settling time. The results imply that the proposed denoising architecture is suitable for real-time denoising applications with acceptable improvement in SNR approximately $8 \mathrm{~dB}$.
\end{abstract}


Keywords: Discrete wavelet transforms; DSP, VLSI architecture; Signal denoising; Wavelet thresholding.

\section{INTRODUCTION}

The discrete wavelet transform (DWT) is denoted as one of the best methods for denoising nonstationary signals. The concept of DWT is based on decomposing the signals into different frequency bands at different scales, due to these properties, the analysis of non-stationary signals become possible. Since most of biomedical signals are considered as non-stationary signals, therefore, the DWT is considered as a good tool for denoising biomedical signals (Samann \& Schanze, 2019)(Wang, Zhu, Yan, \& Yang, 2019). In practical, several researches have suggested that the DWT with lifting scheme has many benefits over the conventional DWT specially in term of realization of the lifting scheme for real-time applications (Daubechies \& Sweldens, 1998) (Acharya \& Tsai, 2005)(Saleh \& Al-Sulaifanie, 2017). In recent years, many researchers have been working on developing wavelet thresholding method to estimate a proper threshold for denoising biomedical signals due to the ability of DWT in providing appropriate basis for separating noise from noisy signal. A real-time VLSI based on DWT denoising method is developed by (Kasambe \& Rathod, 2015) to eliminate the power-line interference from PPG signals. In (Bahoura \& Ezzaidi, 2010), the authors present the implementation of real-time wavelet denoising method on FPGA, and this method is used to remove power-line noise from ECG signals. In the work (Saleh \& Al-Sulaifanie, 2017), a VLSI integrated architecture for computing a 1-D IWT was proposed. The adaptive threshold is estimated by computing the median absolute deviation (MAD) through sorting successively samples of wavelet detail coefficients of the first level. The work in (Ballesteros \& Moreno, 2013) introduces real-time wavelet denoising architecture with adaptive thresholding method. The adaptive thresholding method is developed for real-time sorting applications that based on an insertion algorithm to compute the median of the detail coefficients. The threshold is updated every 8 cycles. 
In (Harrison, 2003), adaptive thresholding detector was developed to estimate the standard deviation of background noise by maintaining the duty-cycle at a required value of $15.9 \%$. This method can be implemented by a low-power analog integrated circuit. The adaptive thresholding algorithm was developed based on the assumption of having spikes added to band-limited white gaussian noise as an input to the system. For a white gaussian noise signal, the probability of a sample having value above standard deviation is 0.159 . A proportional feedback control loop is used to adapt the threshold of comparator until its output has a duty cycle of $15.9 \%$ (Watkins et al., 2004). Wavelet DWT and SWT (stationary wavelet transforms) have been applied to spike detection (Kim \& Kim, 2003) (RJ et al., 2007). A new spike detector is presented by (Yang, Boling, Kamboh, \& Mason, 2015) that use system-on-chip approach to implement adaptive threshold estimation for high accurate spike detection. In the work (Gagnon-Turcotte et al., 2016), adaptive thresholding method based on a sigma-delta control was developed for resource optimized digital action potential detector. Most of the research in the literatures has worked on developing adaptive threshold estimation which was dedicated for biomedical signals such as action potential and ECG detector featuring.

The goal of this paper is to design a real-time wavelet denoising architecture that can be used in wide range applications. The proposed architecture is based on haar wavelet transform with lifting scheme. Besides, an adaptive threshold estimator based on feedback control loop is considered to make the design more suitable for real-time denoising. The proposed architecture is developed and simulated using MATLAB 2018b. The rest of the paper is organized as follows: In section 2, real-time denoising wavelet-based architecture and adaptive thresholding method are described in details. The results and performance of the proposed architecture are stated in section 3. At the end, the conclusion is given in section 4 . 


\section{THE PROPOSED ARCHITECTURE}

The real-time wavelet-based denoising architecture embraces decomposition by DWT, delay alignments, adaptive thresholding and reconstruction by the inverse IDWT. The block diagram of the proposed wavelet-based denoising architecture is shown in figure 1 . The design issues of the proposed architecture take into the account the following consideration. The errorintroduced by decomposition and reconstruction modules should be insignificant. A noise level estimator module will dynamically estimate the unknown standard deviation $S D_{a}$ of the background noise from the first detail level $d_{1}$ of DWT using a feedback control loop. An adaptive threshold estimator calculates the threshold level $T H=S D_{a} \times F$, where $F$ is a constant factor, that to be fixed. The propagation delays between the signals should be alignment at different levels and dynamically compared to the online adaptive threshold using the soft threshold module.

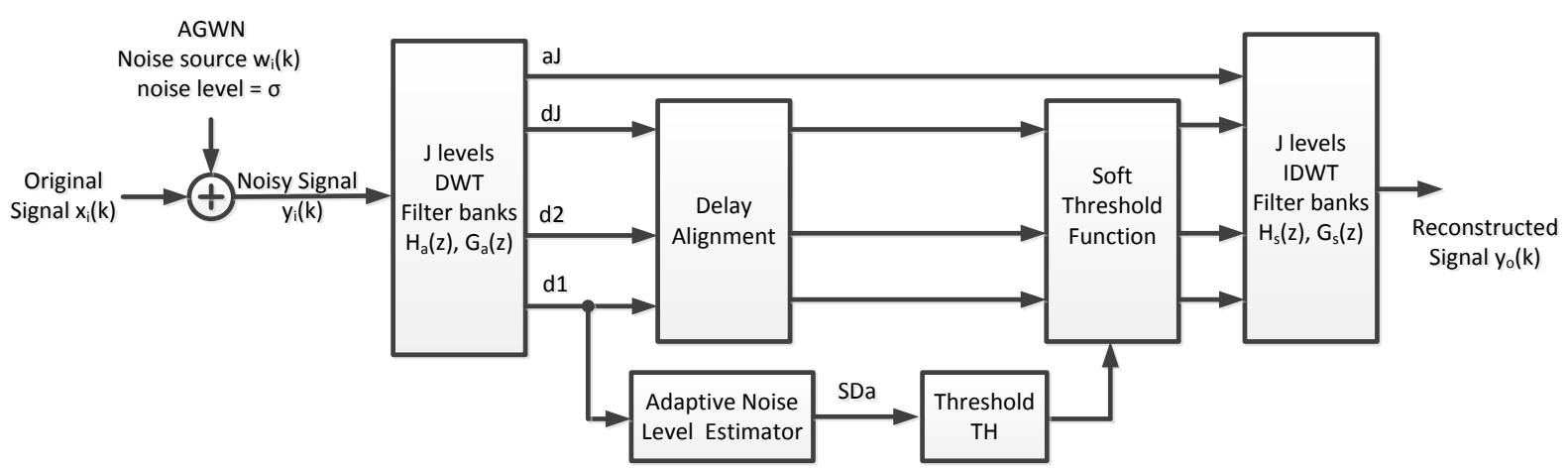

Figure 1 The block diagram of the proposed real-time wavelet-based denoising architecture.

\section{LIFTING SCHEME OF HAAR WAVELET TRANSFORM}

Haar wavelet filter based on lifting scheme is considered in the proposed architecture. Since the lifting scheme approach requires a smaller number of adders, multipliers and shifters, and less computational time comparing with the conventional DWT (Saleh \& Al-Sulaifanie, 2017),so it 
is more suitable for real-time applications. The coefficients of Haar filter banks for the unnormalized analysis and synthesis are given in (Daubechies \& Sweldens, 1998) as follows:

Analysis low pass filter (LPF) $H_{a}(z)=\frac{1}{2}+\frac{1}{2} z^{-1}$,

Analysis high pass filter $(\mathrm{HPF}) G_{a}(z)=-1+z^{-1}$,

Synthesis LPF $H_{s}(z)=1+z^{-1}$, and synthesis $\operatorname{HPF} G_{s}(z)=-\frac{1}{2}+\frac{1}{2} z^{-1}$.

The forward wavelet transform of with Haar filters can be implemented in three steps using lifting scheme as:

a. Splitting: the input signal $x(n)$ at sampling rate $f_{s}$ is split into even $x_{e}(k)=x(2 k)$ and odd $x_{o}(k)=x(2 k-1)$ sequences with half sampling rate $f_{s} / 2$.

b. Prediction: the odd sample is predicted based on the even sample through a predicting operator $\mathrm{P}$ and the prediction detail signal is

$$
\mathrm{d}_{1}(\mathrm{k})=-\mathrm{x}_{\mathrm{e}}(\mathrm{k})+\mathrm{x}_{\mathrm{o}}(\mathrm{k})
$$

c. Updating: the detail signal $\mathrm{d}_{1}(\mathrm{k})$ is update through an updating operator $\mathrm{U}$, to get the approximation signal as

$$
a_{1}(k)=x_{e}(k)+0.5 d_{1}(k)
$$

The decomposition and reconstruction process of lifting scheme Haar wavelet transform is shown in figure 2 . The reconstructed output of the lifting scheme is delayed with the input by one clock. 


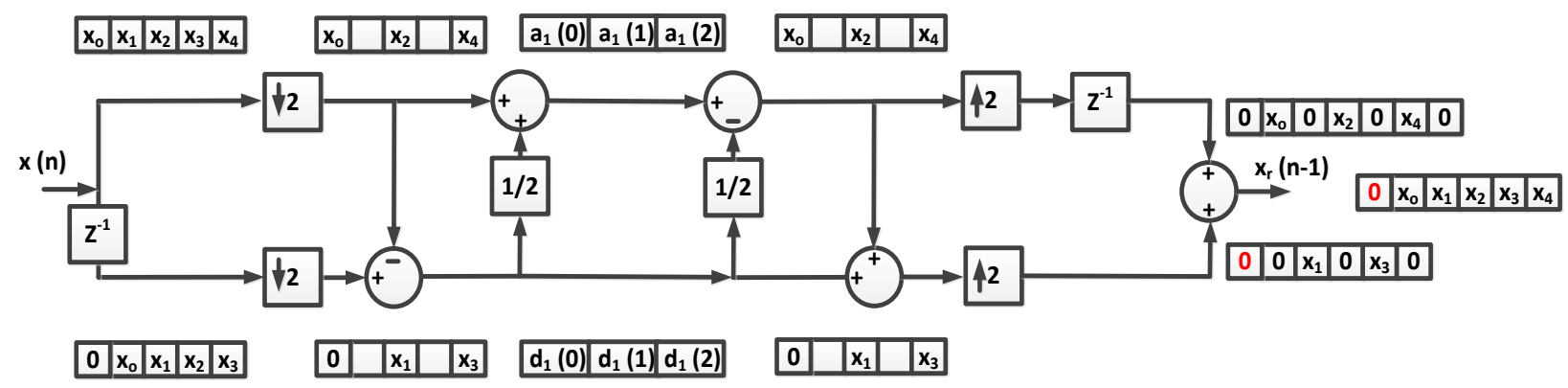

Figure 2 Decomposition and Reconstruction process of lifting scheme Haar wavelet transform

The reconstruction process is achieved by simply inverting the direction of data flow and operators in the original formula, then merging process is considered instead of splitting. The even sample can be computed as $\mathrm{x}_{\mathrm{e}}(\mathrm{k})=\mathrm{a}(\mathrm{k})-\frac{1}{2} \mathrm{~d}(\mathrm{k})$. While the odd samples are $\mathrm{x}_{\mathrm{o}}(\mathrm{k})=$ $\mathrm{d}(\mathrm{k})+\mathrm{x}_{\mathrm{e}}(\mathrm{k})$

In general, the filter of length $\mathrm{L}$ and for J level of DWT decomposition/reconstruction the delay between input and reconstructed output at decomposition level J can be computed according to following formula

$$
\mathrm{DF}_{\mathrm{j}}=(\mathrm{L}-1)\left(2^{\mathrm{J}-\mathrm{j}}-1\right)
$$

For Haar filter banks, each filter length is $\mathrm{L}=2$. For three levels of transformation $\mathrm{J}=3$, the introduced delays $D F_{j}$ of the details coefficient outputs $d_{j}$ are $D F_{1}=3$ at first level, while $\mathrm{DF}_{2}=1$, and $\mathrm{DF}_{3}=0$ for levels two and three respectively. While the total delay between the reconstructed output and the input is $\mathrm{DF}_{0}=7$.

\section{ADAPTIVE NOISE LEVEL ESTIMATOR MODULE}

The noise level from unknown signal is estimated by computing the median absolute deviation (MAD) from the first level detail coefficients $d_{1}(n)$ to compute the standard deviation as follows (Donoho \& Johnstone, 1995). 


$$
\sigma=\mathrm{MAD} / 0.6745
$$

where MAD $=$ median $\left(\left|d_{1}(n)\right|\right)$ is the median absolute deviation of the wavelet coefficients, estimated on level one $(\mathrm{J}=1)$. Then, the universal threshold $\mathrm{TH}$ is computed using the formula

$$
\mathrm{TH}=\sigma \sqrt{2 \ln (\mathrm{N})}
$$

where $N$ is the length of the $d_{1}(n)$. This method is generally used for off-line processing. Since it is post-processes of the whole signal to detect local events, therefore it is not suitable for realtime applications. Furthermore, in case of the standard deviation of the noise is not being stationary, it requires repetition of the measurements or considering adaptive threshold scheme which is cable of tracking the level of the noise and set the threshold accordingly. A block diagram of the real time noise standard deviation estimator module capable of tracking the noise level is presented in figure 3 . The detail input $d_{1}(n)$ has frequency rate $f_{s} / 2$ with sampling period $2 \mathrm{~T}_{\mathrm{s}}$. The module estimates adaptively the standard deviation value $\mathrm{SD}_{\mathrm{a}}$, of the noise using a feedback control loop. A divided by M target counter is free running counter with input clock frequency $f_{s} / 2$, where $M$ is the number of samples within a window size period of $T_{M}=2 M_{s}$. This counter periodically counts from 0 to $M-1$, within period $T_{M}$. During the period $T_{M}$, the input $d_{1}(n)$ is compared to the previous estimated feedback noise level $S_{a}$ by a loop comparator. At any time, the output of the loop comparator is $\operatorname{LC}(n)=1$, when $d_{1}(n)$ is greater than $\mathrm{SD}_{\mathrm{a}}$, otherwise $\mathrm{LC}(\mathrm{n})=0$. The $\mathrm{LC}(\mathrm{n})$ output is gated through logic AND operation with the input clock. The AND gated output clocks are counted by an event counter. The final count of the event counter at the end of the period $\mathrm{T}_{\mathrm{M}}$ is denoted as $\mathrm{N}_{\mathrm{E}}$. The instant error signal $\mathrm{e}$ between the count of the two counters is given by:

$$
\mathrm{e}(\mathrm{n})=\mathrm{E}_{\mathrm{CO}}(\mathrm{n})-0.159 \mathrm{~T}_{\mathrm{CO}}(\mathrm{n})
$$


Where $E_{C O}(n)$ and $T_{C O}(n)$ are the corresponding instant counting of the Event and Target counter respectively. At the end of each period $\mathrm{T}_{\mathrm{M}}$, the target counter count $\mathrm{M}$ samples, and the event counter count the number of the detail samples $\mathrm{N}_{\mathrm{E}}$, that are greater than $\mathrm{SD}_{\mathrm{a}}$. $\mathrm{A}$ synchronized carry ripple out $\mathrm{C}_{\mathrm{r}}(\mathrm{n})$ from the target counter is activated whenever its count is $M-1$. The output $C_{r}(n)$ is used to capture and store the ending values of the error signal e(n) at each period $\mathrm{T}_{\mathrm{M}}$ in a register. This mean that a multi-rate foundation with down sampling $\mathrm{f}_{\mathrm{s}} / \mathrm{M}$ is achieved. Then the output of the register can be expressed as:

$$
\mathrm{e}(\mathrm{Mn})=\mathrm{E}_{\mathrm{CO}}(\mathrm{Mn})-0.159 \mathrm{~T}_{\mathrm{CO}}(\mathrm{Mn})=\mathrm{N}_{\mathrm{E}}-0.159 \mathrm{M}
$$

It is known from the Gaussian distribution theory that the probability of the level of the Gaussian noise signal exceeds its standard deviation $\sigma$ is $15.9 \%$. Therefore, at the steady-state condition of the error signal e(Mn) will approach zero, whenever the event count $\mathrm{N}_{\mathrm{E}}$ is close to $0.159 \mathrm{M}$. A feedback control digital integral $\mathrm{C}(\mathrm{z})$ is used to integrate the signal $\mathrm{e}(\mathrm{Mn})$, and the output of the $\mathrm{C}(\mathrm{z})$ represent the estimated adaptive standard deviation $\mathrm{SD}_{\mathrm{a}}$. Under stable conditions, the $\mathrm{e}(\mathrm{Mn})$ will vary slightly up and down around zero value, and the count $\mathrm{N}_{\mathrm{E}}$ maintained close to $\mathrm{M} * 0.159$, when $\mathrm{SD}_{\mathrm{a}} \approx \sigma$. The noise estimator module computes and continually updates $\mathrm{SD}_{\mathrm{a}}$ values at end of each period $\mathrm{T}_{\mathrm{M}}$ of the time, registering its result when $\mathrm{M}$ samples have elapsed. The output of the register $\mathrm{e}(\mathrm{Mn})$ is integrated every $\mathrm{T}_{\mathrm{M}}$ cycle. The transfer function of the digital integrator is

$$
C(z)=G \frac{1}{\left(1-z^{-1}\right)}
$$

where $\mathrm{G}$ is the gain of the integrator. The timing diagram of the standard deviation estimator are presented in figure 4 for $T_{s}=1, \sigma=2, M=64, G=1 / 16$. 


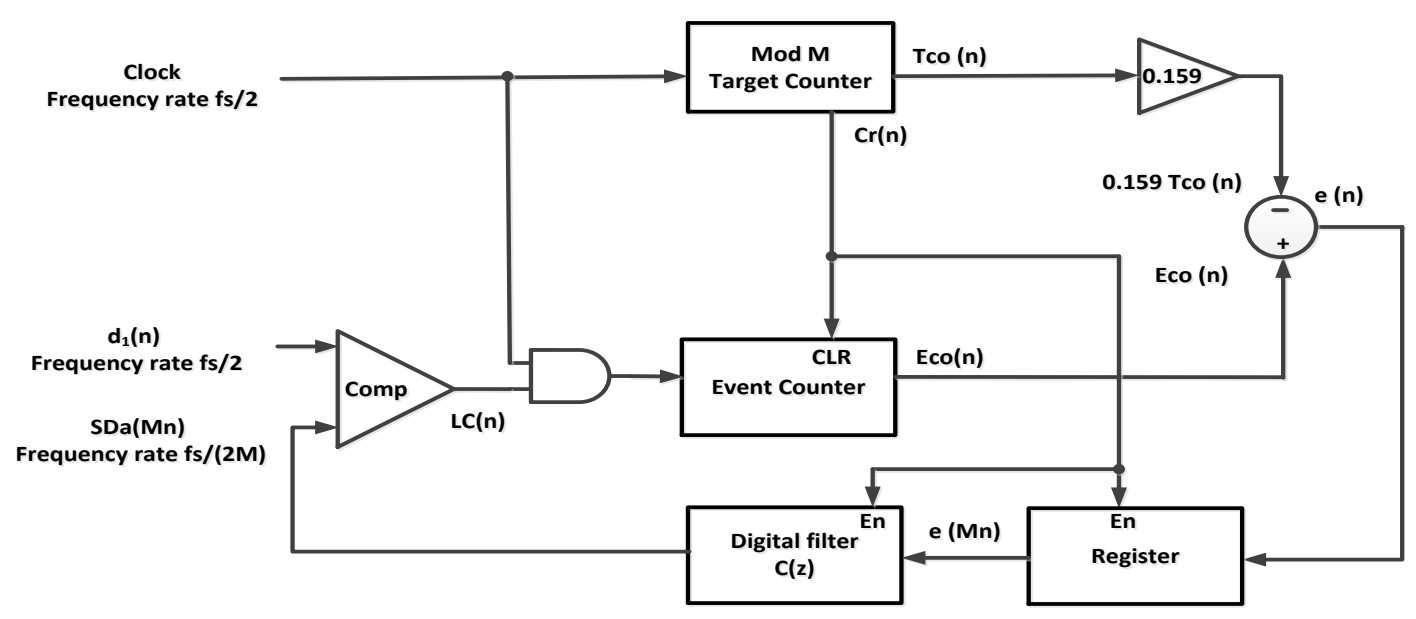

Figure 3 The block diagram of the standard deviation estimator.

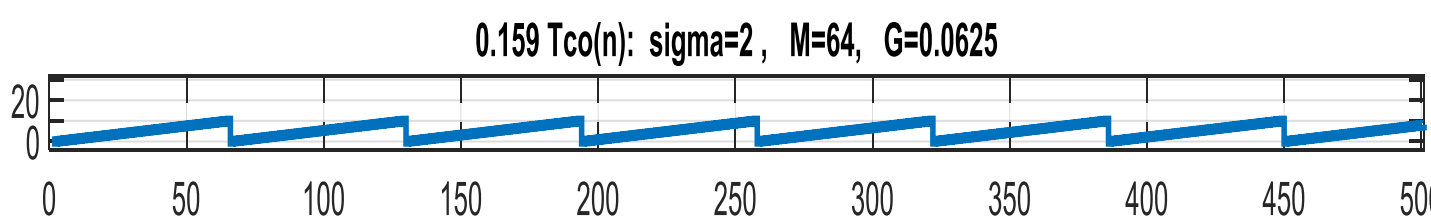

Cr(n)

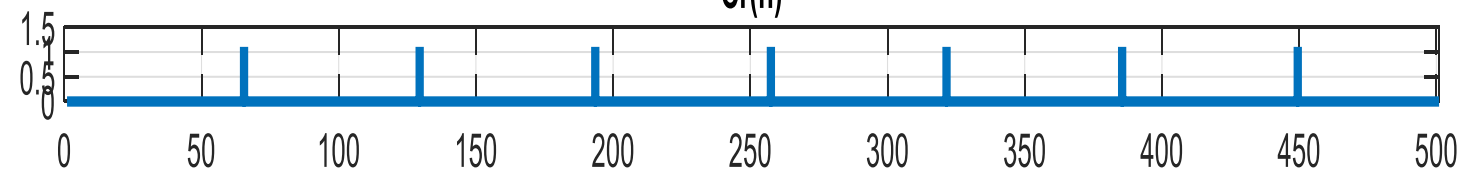

Eco(n)

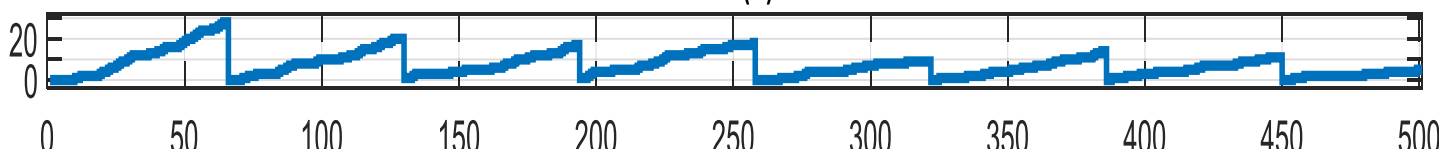

$e(n)$

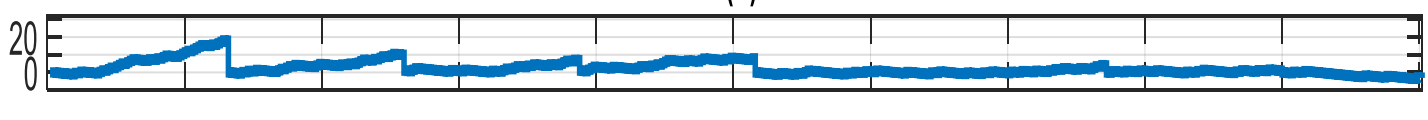

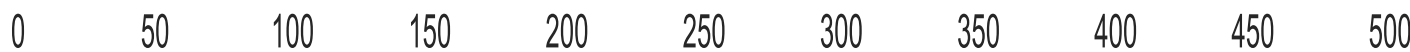

$\mathrm{e}(\mathrm{Mn})$
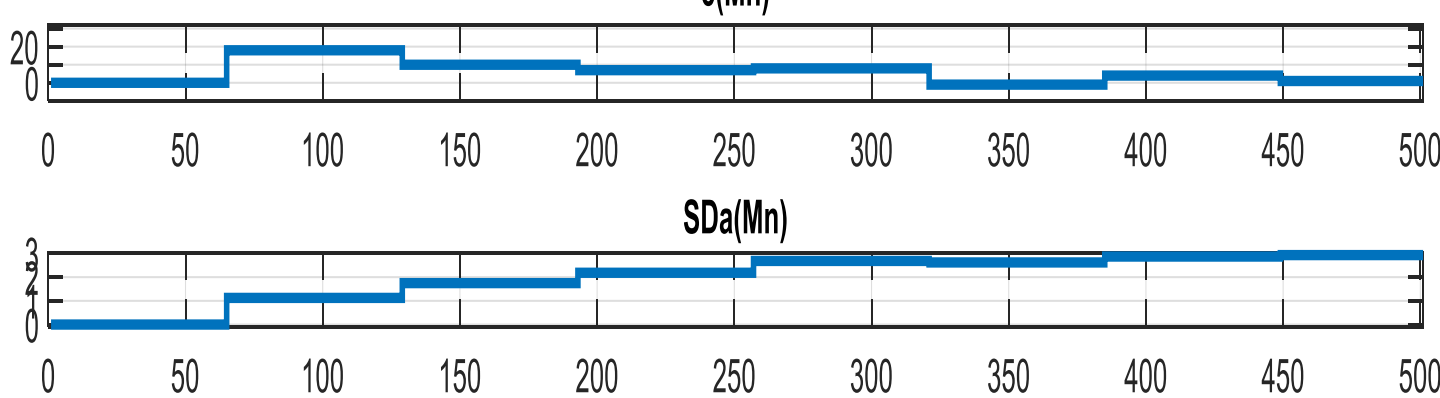

Figure 4 The timing diagram of the standard deviation estimator module. 


\section{THRESHOLD ESTIMATOR MODULE}

The estimation of threshold value plays significant role in the performance of wavelet-based denoising approach. Therefore, several methods of threshold value estimation have been proposed. Among these existing methods, the most popular one is universal threshold. This is because the probability of noise amplitude being just below the universal threshold $T H$ is very high. The universal threshold is proposed by (Donoho and Johnstone, 1995) which depends on the estimated noise level and the length of the samples in the window size. In the proposed scheme, the threshold is calculated adaptively as follows:

$$
T H=S D_{a} \times F=S D_{a} \sqrt{2 \ln (M)}
$$

Where $S D_{a}$ is the updated real time estimated standard deviation of the noise within each window size of $M$ samples sequentially from the detail input $d_{1}(n)$. The multiplier factor by $S D_{a}$ in (9) is defined as $F=\sqrt{2 \ln (M)}$, which can be predetermined as function of $M$ as shown in table 1. For a white Gaussian noise, most of the maximum and minimum samples are lies between $-3 \sigma$ and $3 \sigma$ (about 99.73\%). So, setting the threshold above and below this two ranges, ensures the cancellation of the almost noisy coefficients of $d_{j}(n)$.

Table 1 Multiplier factor $F$ as function of $M$

\begin{tabular}{|l|l|l|l|l|l|l|l|}
\hline $\boldsymbol{M}$ & 8 & 16 & 32 & 64 & 128 & 256 & 1024 \\
\hline $\boldsymbol{F}$ & 2.0393 & 2.3548 & 2.6328 & 2.8841 & 3.1151 & 3.3302 & 3.7233 \\
\hline
\end{tabular}

For the three levels of decomposition $(J=3)$, the estimated $\mathrm{TH}$ is used to threshold the detail signals $d_{1}, d_{2}$, and $d_{3}$. However, due to multi-rate behavior of the signals $d_{1}, d_{2}$, and $d_{3}$ the threshold level TH should be down sampled by 2 and 4 for the detail signals, $d_{2}$, and $d_{3}$ 
respectively, in order to maintain the same sampling rate at different levels of wavelet decomposition.

\section{SOFT THRESHOLD MODULE}

After calculating the threshold, the detail coefficients $d_{j}(n)$ are replaced with zero when they are not exceeding the threshold value regarding to the following soft threshold rule (ZHANG, LU, \& LIU, 2019):

$$
d_{j T}(\mathrm{n})=\left\{\begin{array}{cc}
d_{j}(n)-T H & \text { if } d_{j}(n)>T H \\
d_{j}(n)+T H & \text { if } d_{j}(n)<-T H \\
0 & \text { if }\left|d_{j}(n)\right| \leq T H
\end{array}\right.
$$

Where $d_{j T}(\mathrm{n})$ is the thresholded detail coefficients.

The complete block diagram outline of the proposed real-time wavelet-based denoising architecture is shown in figure 5.

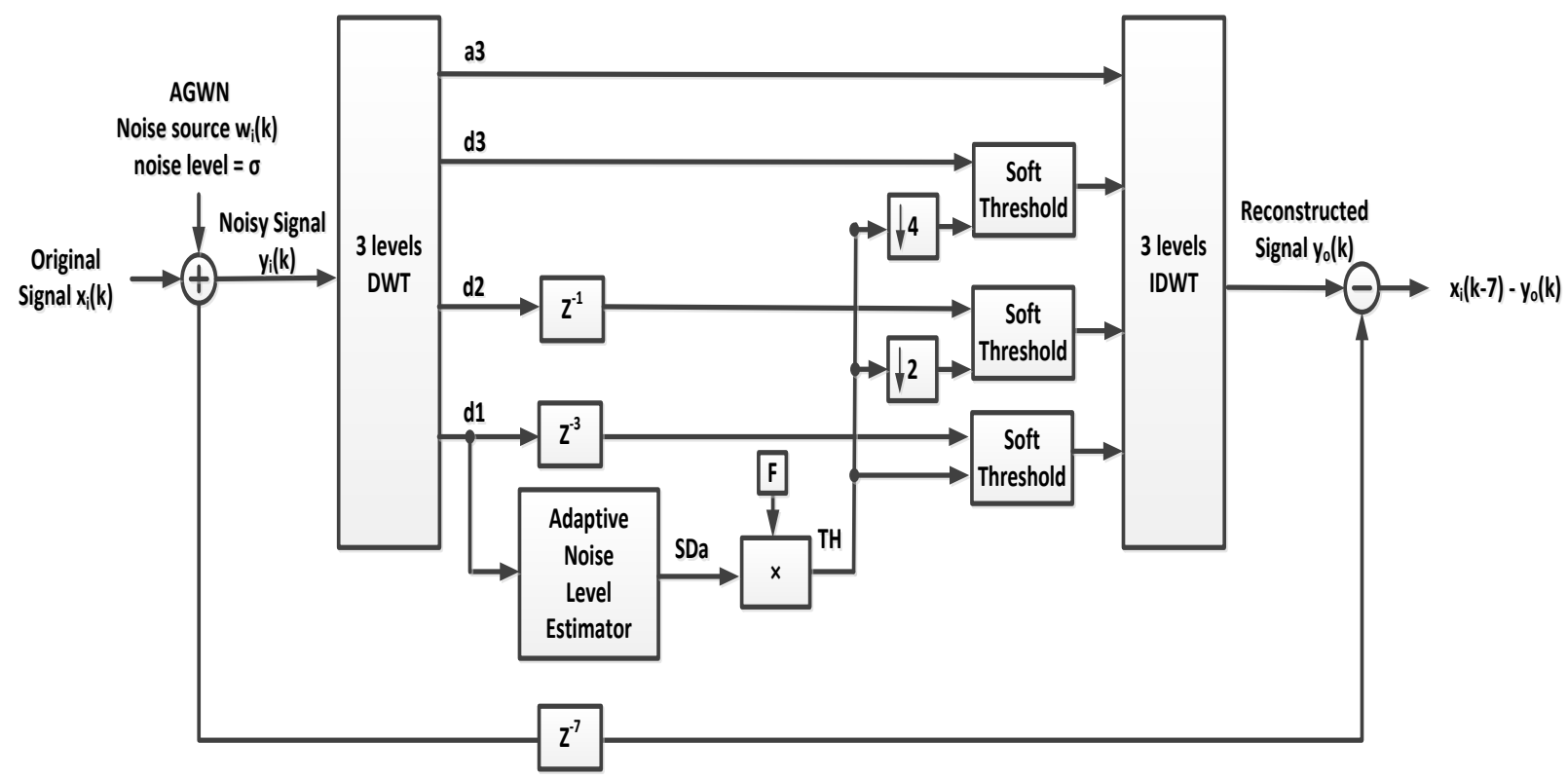

Figure 5 Block diagram of the proposed real-time denoising wavelet-based architecture 


\section{RESULTS AND DISCUSSION}

The performance of the proposed real-time wavelet denoising method is evaluated by denoising the corrupted rectangular pulse signal $y_{i}(n)$. The corrupted signals of different input SNR levels $(-5 \mathrm{~dB}, 0 \mathrm{~dB}, 5 \mathrm{~dB}$ and $10 \mathrm{~dB})$ are generated by adding simulated gaussian white noise $w_{i}(n)$ to the original signal $x_{i}(n)$ as shown in figure 6 . The following specifications of $x_{i}(n)$ are considered fundamental frequency $f_{o}=0.001 \mathrm{~Hz}$ and the normalized sampling frequency $f_{s}=$ $1 \mathrm{~Hz}$, and amplitude equal to 5 volts. The denoised output signal $y_{o}(k)$ is reconstructed after three levels of wavelet decomposition/reconstruction using adaptive soft thresholding method. The measurement criteria signal-to-noise $S N R$ and mean square error $M S E$ parameters are considered to evaluate the performance. The criteria measurement input $S N R_{I}$ and output $S N R_{o}$ are calculated as follow:

$$
S N R_{I}=10 \log _{10}\left(\frac{\sigma_{x_{i}}^{2}}{M S E_{i}}\right) \quad d B
$$

Where is $\sigma_{x_{i}}^{2}$ is the variance or AC power of the original input signal $x_{i}(k)$, The mean-squareerror $M S E_{i}$ is denoted as follow.

$$
M S E_{i}=\sigma^{2}=\frac{1}{N} \sum_{k=0}^{N-1}\left(x_{i}(k)-y_{i}(k)\right)^{2}
$$

Where $N$ is the length of input the signal. The quality of the output denoised signal $y_{o}(k)$ is measured by the output $S N R_{O}$ and $M S E_{o}$ as:

$$
\begin{aligned}
& S N R_{o}=10 \log _{10}\left(\frac{\sigma_{x_{i}}^{2}}{M S E_{o}}\right) d B \\
& M S E_{o}=\frac{1}{N} \sum_{k=0}^{N-1}\left(x_{i}(k)-y_{o}(k)\right)^{2}
\end{aligned}
$$



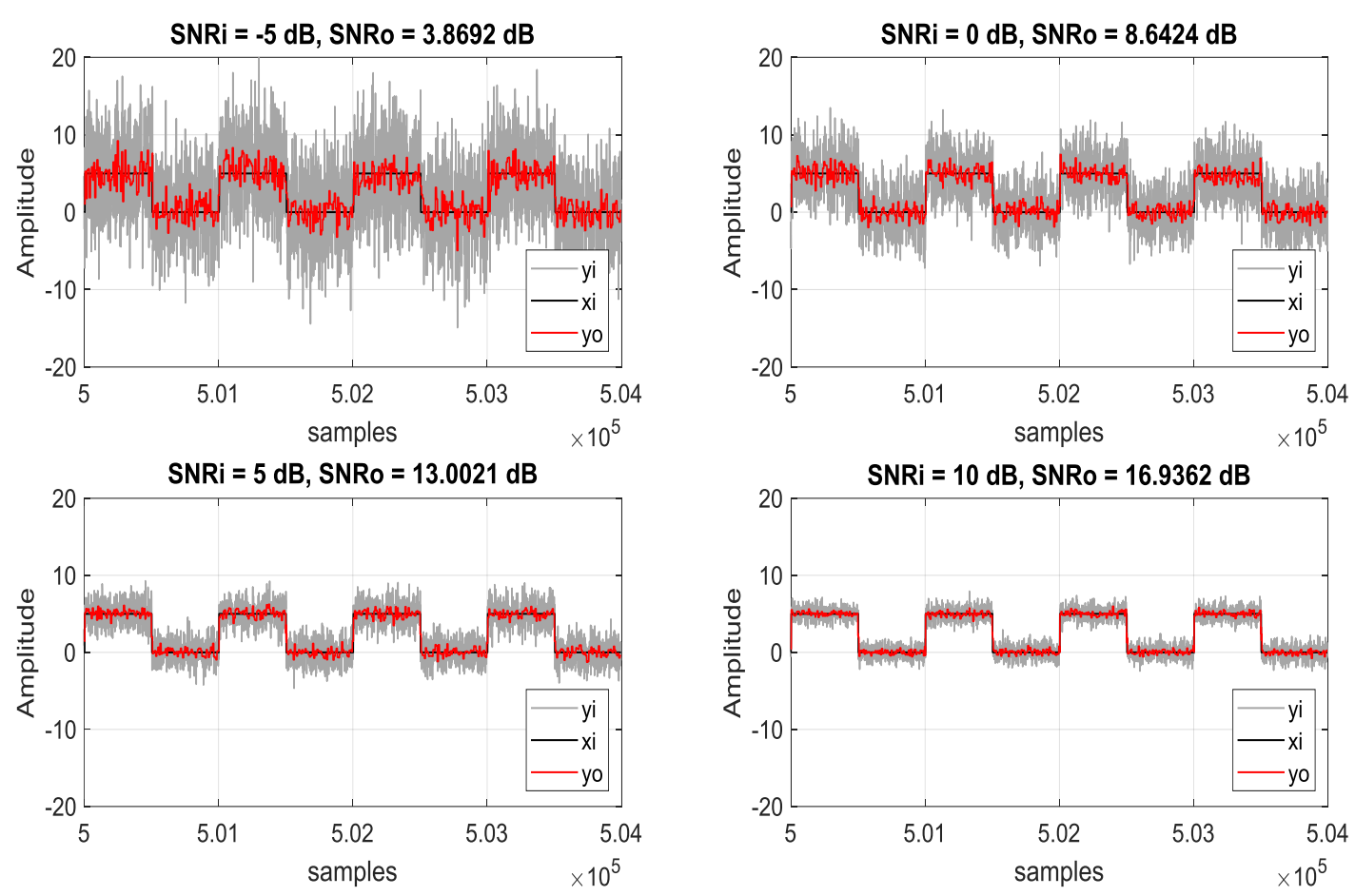

Figure 6 The rectangular pulse waveform $x_{i}(n)$ and the noisy signal $y_{i}(n)$ and reconstructed denoised signal $y_{o}(k)$ at (upper left) $S N R_{I}=-5 \mathrm{~dB}$ (upper right) $S N R_{I}=0 \mathrm{~dB}$ (lower left) $S N R_{I}=$ $5 \mathrm{~dB}$ (lower right) $S N R_{I}=10 \mathrm{~dB}$. The gain $G$ and window size $M$ are chosen to be $G=1 / 64$ and $M=64$ based on a study

The performance of the proposed denoising method is studied in terms of integral gain $G$ of feedback control and window size $M$ with respect to the improvement in SNR and settling time. To select the best values of $G$ and $M$, the performance of the proposed method is investigated by varying gain $G$ and kept the window size $M$ fixed for different cases of $M$ as shown in figure 7 and 8 . In general, the results show that small values of window size $M(M=16 \& M=64)$ always has better improvement in term of SNR especially in case of large values of $G$ (like $G=1 / 16$ and $G=1 / 64$ ) (see figure 7). However, the large $M$ gives more accurate estimation of the $S D_{a}$ noise at the cost of longer settling time (see figure 8). Moreover, it is clearly seen in figure 8 that small values of $G$ (like $G=1 / 256$ and $G=1 / 1024$ ) require longer time to reach the final response of $S D_{a}$ noise. Since the estimated $S D_{a}$ is directly linked to the thresholding method (see Equation 9), this delay in estimation causes degradation in denoising the signal in term of SNR but more 
accurate and stable estimation of $S D_{a}$ as shown clearly in figure 9. Besides, in real-time applications, it is better to have smaller window size $M$ and smaller settling time. In this design, the value of $M$ and $G$ are chosen to be 64 and 1/64 respectively by considering the trade-off between the improvement of SNR and settling time. In figure 6, It can be clearly observed that a significant amount of noise has been removed. In case of $S N R_{I}=0 \mathrm{~dB}$, a massive noise is suppressed, and the outlook frame of the original signal is recovered with maximum improvement of $8.6424 \mathrm{~dB}$. When the $S N R_{I}$ equal to $10 \mathrm{~dB}$, the original signal is entirely recovered with minor distortion. However, in case of $S N R_{I}=10 \mathrm{~dB}$, the mount of improvement in term of signal-to-noise ratio is only $6.9362 \mathrm{~dB}$.
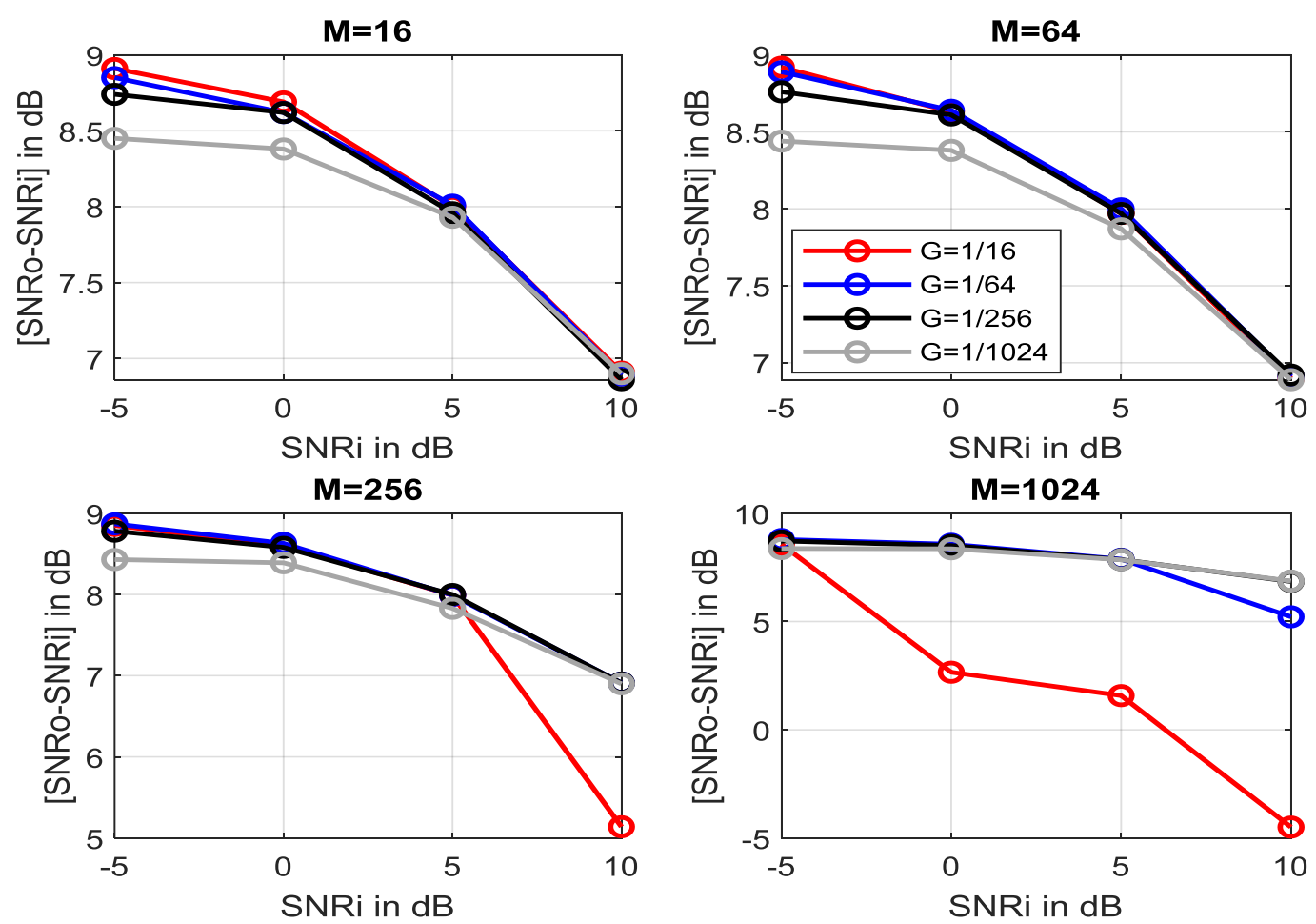

Figure 7 The performance of the proposed denoising method in term of improved signal-to-noise ratio when the window size of $M$ is fixed, and the gain $G$ is varied. 

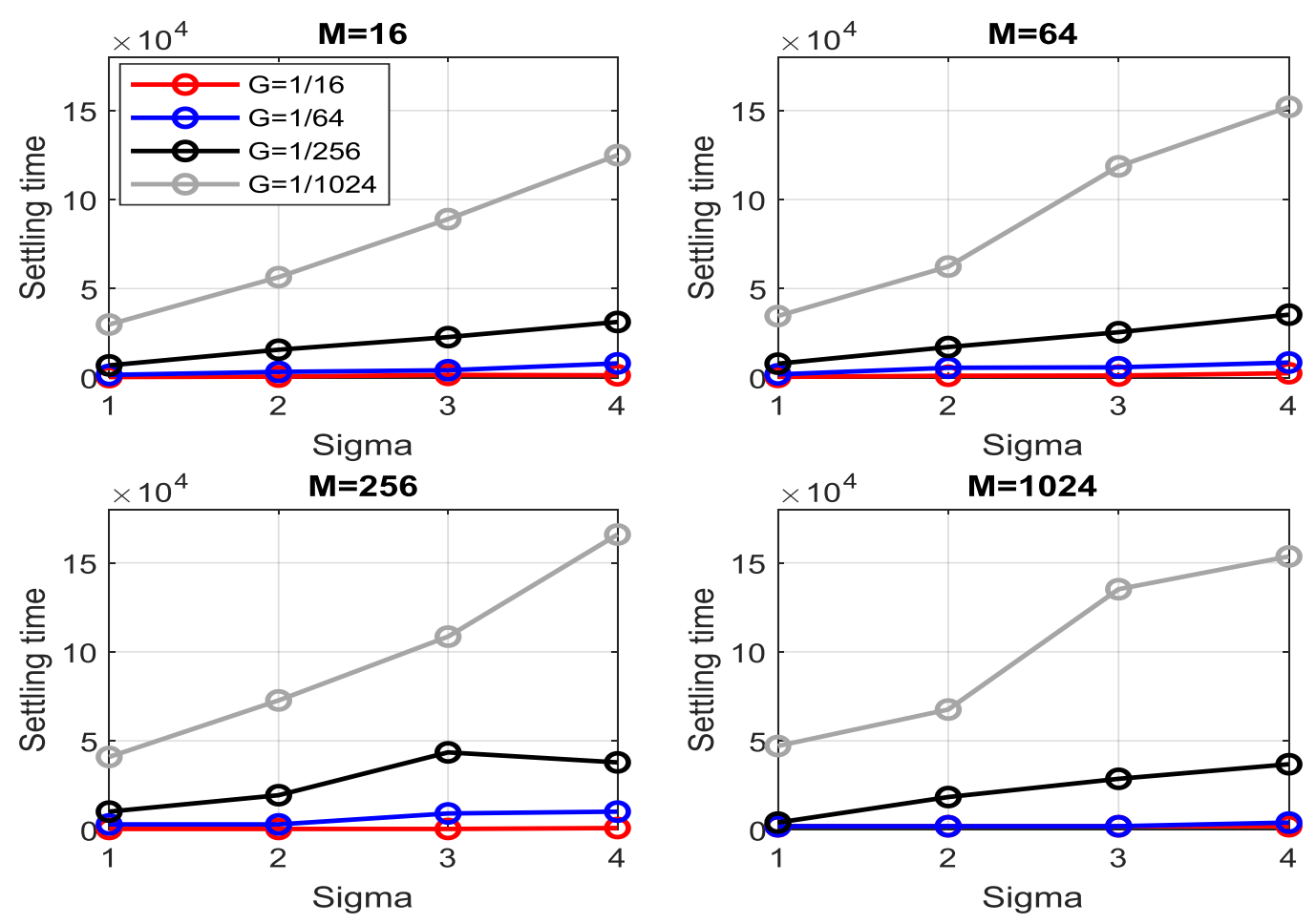

Figure 8 The performance of the proposed denoising method in term of setline time for different levels of noise sigma when the window size of $M$ is fixed, and the gain $G$ is varied.

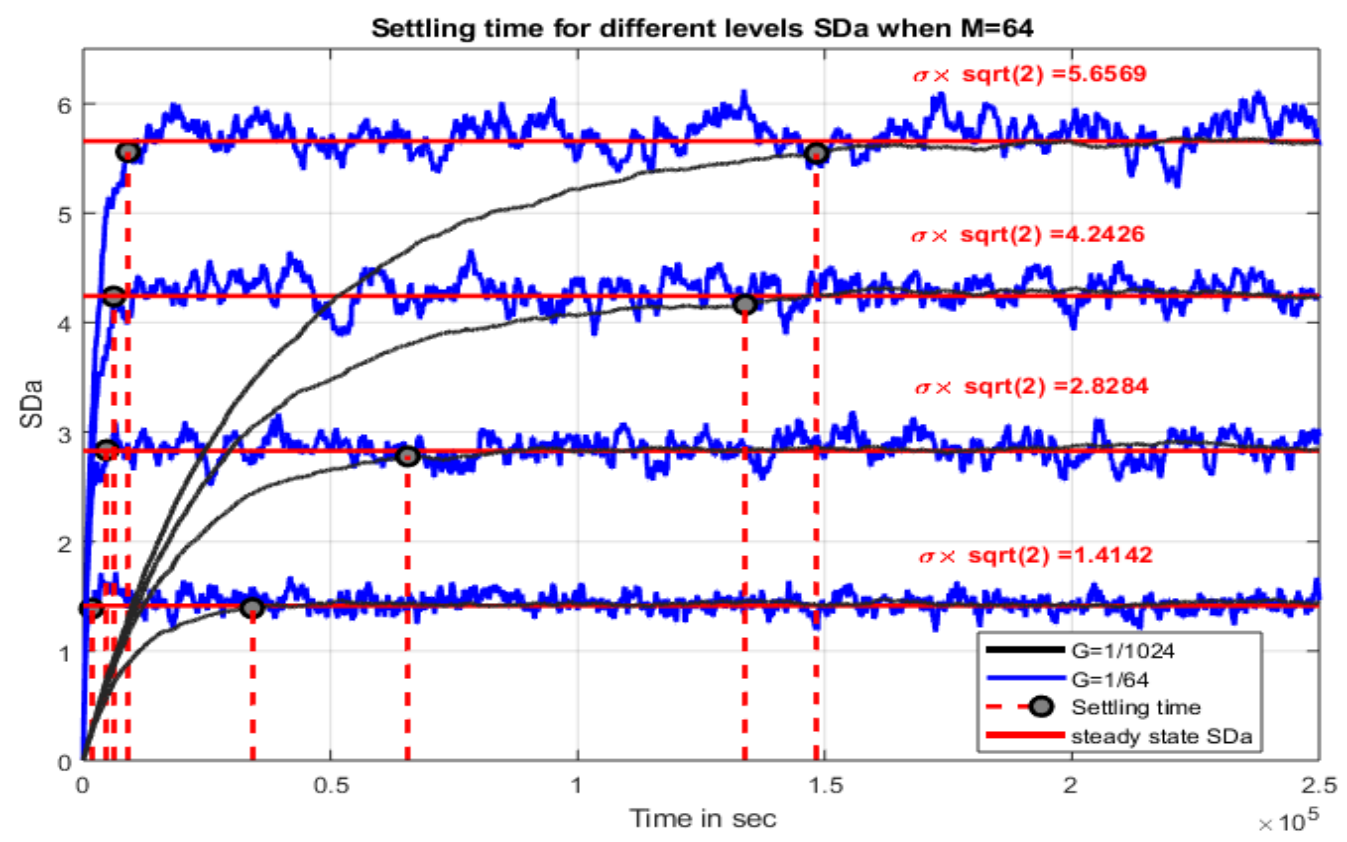

Figure 9 The setting time required to reach the final value (steady state) of the estimated $S D_{a}$ for $G=1 / 1024$ and $G=1 / 64$ with $M=64$ 


\section{CONCLUSIONS}

Adaptive real-time wavelet denoising architecture based on feedback control loop is developed in this paper. Three levels of DWT with harr lifting scheme is considered. In real-time denoising, estimating the threshold value for wavelet coefficients plays essential role in denoising the signal. In this architecture, adaptive thresholding method is developed using a noise level estimator module based on first detail coefficients level $d_{1}$ with integral feedback control system to estimate the unknown standard deviation $S D_{a}$ of background noise. The performance of feedback control system in estimating the $S D_{a}$ is investigated in term of settling time and improved SNR. The results show that the selection of gain $G$ and window size $M$ has a significant impact on the convergence time (settling time) and the accuracy in estimating $S D_{a}$ of the noise. The trade-off between the accuracy and settling time in estimating $S D_{a}$ should be made regarding to the requirements of the application. Regardless of the fluctuation and inaccuracy in the estimated $S D_{a}$ considering $M=64$ and $G=1 / 64$, the average improvement in term of SNR is interestingly high approximately $8 \mathrm{~dB}$ (see figure 6). Besides, the time required for estimating the noise standard deviation level is suitable for real-time application to calculate the adaptive threshold. In the future, the proposed denoising architecture will be tested on real biomedical signals and implemented on FPGA configurable digital integrated circuit for online applications.

\section{REFERENCES}

Acharya, T., \& Tsai, P.-S. (2005). JPEG2000 Standard for Image Compression Concepts, Algorithm and VLSI Architectures. New Jersey: John Wiley \& Sons.

Bahoura, M., \& Ezzaidi, H. (2010). FPGA-implementation of waveletbased denoising technique to remove power-line interference from ECG signal. Proceedings of the 10th IEEE International Conference on Information Technology and Applications in Biomedicine. Corfu. 
Ballesteros, D. M., \& Moreno, J. M. (2013). Wavelet-denoising on hardware devices with Perfect Reconstruction, low latency and adaptive thresholding. Computers \& Electrical Engineering, 39(4), 1300-1311.

Daubechies, I., \& Sweldens, W. (1998). Factoring wavelet transforms into lifting steps. Journal of Fourier Analysis and Applications, 4, 247-269.

Donoho, D. L., \& Johnstone, M. I. (1995). Adapting to unknown smoothness via wavelet shrinkage. Journal of the American Statistical Association December, 90(432), 1200-1224.

Gagnon-Turcotte, G., LeChasseur, Y., Bories, C., De Koninck, Y., \& Gosselin, B. (2016). An optimized adaptive spike detector for behavioural experiments. IEEE Internationnal Symposium On Circuits and Systems (ISCAS'16). Montreal, QC, Canada.

Harrison, R. R. (2003). A Low-Power Integrated Circuit for Adaptive Detection of Action Potentials in Noisy Signals. Proceedings of the 2Sth Annual International Conference of the IEEE EMBS, 3325-3328. Cancun, Mexico.

Kasambe, P. V, \& Rathod, S. S. (2015). VLSI Wavelet Based Denoising of PPG Signal. Procedia Computer Science, 49, 282-288. https://doi.org/10.1016/j.procs.2015.04.254

Kim, K. H., \& Kim, S. J. (2003). A wavelet-based method for action potential detection from extracellular neural signal recording with low signal-to noise ratio. IEEE Transactions on Biomedical Engineering, 50(8), 999-1011.

RJ, B., S, T., M, A., NR, K., D, R., RG, S., \& A., D. (2007). Wavelet methods for spike detection in mouse renal sympathetic nerve activity. IEEE Transactions on Biomedical Engineering, 54(1), 82-93.

Saleh, A. N., \& Al-Sulaifanie, A. K. (2017). REAL TIME VLSI IWT DENOSING ARCHITECTURE. Journal of University of Duhok, 20(1), 164-174. 
Samann, F., \& Schanze, T. (2019). An efficient ECG Denoising method using Discrete Wavelet with Savitzky-Golay filter. 53rd Annual Conference of the German Society for Biomedical Engineering. Frankfurt, Germany.

Watkins,P.T., Santhanam,G.,Shenoy, K. V., andHarrison,R.R.(2004). "Validation of adaptive threshold spike detector for neural recording" Engineering in Medicine and BiologySociety, 2004, IEMBS‘04, in $26^{\text {th }}$ Annual International Conference of the IEEE, Vol.2,SanFrancisco,CA, 4079-4082.

Wang, Z., Zhu, J., Yan, T., \& Yang, L. (2019). A new modified wavelet-based ECG denoising. $\begin{array}{llll}\text { Computer } & \text { Assisted } & \text { Surgery, } & \text { 24(1), }\end{array}$ https://doi.org/10.1080/24699322.2018.1560088

Yang, Y., Boling, C. S., Kamboh, A. M., \& Mason, A. J. (2015). Adaptive Threshold Neural Spike Detector Using Stationary Wavelet Transform in CMOS. IEEE Transactions on Neural Systems And Rehabilitation Engineering, 23(6), 946-955.

ZHANG, M., LU, C., \& LIU, C. (2019). Improved double-threshold denoising method based on the wavelet transform. OSA Continuum, 2(8), 2328-2342. 